\title{
Vigilancia ambiental de casos esporádicos de legionelosis en el municipio de Córdoba (España), 2005-2010
}

\author{
Ana RUBIO GARCÍA \\ Servicio de Salud Pública \\ Distrito Sanitario Córdoba \\ Servicio Andaluz de Salud \\ ana.rubio.sspa@juntadeandalucia.es \\ Joaquín GÁMEZ DE LA HOZ \\ Servicio de Salud Pública \\ Distrito Sanitario Costa del Sol (Málaga) \\ Servicio Andaluz de Salud \\ jgamez@andaluciajunta.es
}

Recibido: 1 de septiembre de 2014

Enviado a evaluar: 10 de septiembre de 2014

Aceptado: 20 de octubre de 2014

\section{RESUMEN}

Examinamos la información del sistema de vigilancia de la legionelosis en el municipio de Córdoba (España) como recurso preventivo para la gestión en salud ambiental y se describen las implicaciones en salud pública de la investigación de casos esporádicos. Un equipo multidisciplinar analizó la información sobre vigilancia de instalaciones potencialmente contaminadas por Legionella y los datos clínicos de los pacientes calificados como casos comunitarios; mapeando el domicilio de los enfermos usando Sistemas de Información Geográfica (SIG). Legionella pneumophila serogrupo 1 fue identificada en 31 casos esporádicos. Se investigaron 53 fuentes sospechosas, mayoritariamente torres de refrigeración. En ningún caso se logró encontrar el origen de la infección aunque se evidenciaron sistemas pobremente mantenidos, deficiencias estructurales y fallos operacionales. Se identificaron dos áreas geográficas donde se concentran los casos en un radio menor a 500 metros. El hallazgo de dos zonas urbanas sospechosas aconseja reorientar la estrategia preventiva en salud pública.

Palabras clave: Legionella pneumophila; Riesgo; Vigilancia; Sistemas de Información Geográfica; Salud Ambiental; Salud Pública (Fuente: DeCS). 


\title{
Environmental surveillance of sporadic community-acquired cases of Legionnaires' disease in Cordoba city (Spain), 2005-2010
}

\begin{abstract}
We examined the information of legionellosis surveillance system in the municipality of Cordoba (Spain) as a preventive resource for environmental health management and describes the public health implications of sporadic cases investigations. A multidisciplinary team analyzed information on surveillance of water sites potentially contaminated by Legionella and clinical data of the patients classified like community case; mapping patient's home address using geographic information systems (GIS). Legionella pneumophila serogroup 1 was identified in 31 sporadic cases. 53 suspected sources, mainly cooling towers have been investigated. In no event is managed to locate infection source even if poorly maintained systems, structural deficiencies and operational failures were apparent. The use of GIS allowed us to identify two geographic areas where cases are concentrated within one radius of less than 500 meters. The finding of two suspected urban advised to reorient the preventive strategy in public health.
\end{abstract}

Key words: Legionella pneumophila; Risk; Surveillance; Geographic Information Systems; Environmental health; Public health (Source: DeCS).

\section{Surveillance environnementale des cas sporadiques de légionellose dans la municipalité de Córdoba (Espagne), 2005-2010}

\section{RÉSUMÉ}

Analyse et description des implications en santé publique de la surveillance environnementale réalisée dans la recherche de cas esporádicos de milieu communautaire par legionelosis (LD). Une équipe multidisciplinaire s'a chargé d'obtenir l'information clinique des patients; mapeado du domicile des malades en utilisant GIS, identifier des sources suspectes d'infection et mener à terme des inspections d'installations potentiellement contaminées par Legionella. Legionella pneumophila serogrupo 1 a été détecté moyennant test d'antígeno urinario en 31 cas esporádicos de Legionelosis. L'usage de GIS a permis identifier deux zones géographiques où ils se groupent les cas dans un radio moindre à 500 mètres. Ils s'ont recherchés 53 sources suspectes, majorité tours de réfrigération. S'evidenciaron systèmes pobremente maintenus, déficiences structurales et fautes operacionales. Dans aucun cas s'a remporté trouver l'origine de l'infection. La trouvaille de deux zones urbaines conflictuelles où ils se concentrent les cas communautaires il suggère une réorientation de la stratégie préventive en santé publique.

Most clés: Legionella pneumophila; risque; surveillance; Systèmes d'information géographique; Santé de l'environnement; De la santé publique (source: MeSH).

\section{INTRODUCCIÓN}

Una de las prioridades de la Unión Europea para reducir los efectos dañinos en la salud humana relacionados con factores ambientales es prevenir los riesgos asociados con determinadas instalaciones en la transmisión de enfermedades como la legionelosis (CEE,2004).

Legionella pneumophila serogrupo 1 (Lp1) es la bacteria responsable de la mayoría de los casos de enfermedad del Legionario notificados en España (Cano R et 
al. 2001), que cursa como neumonía aguda severa transmitida principalmente por aspiración y aerosolización de agua contaminada por Legionella. Los factores de riesgo asociados con esta enfermedad comprenden el consumo de tabaco, edad superior a 50 años, sexo masculino y enfermedades subyacentes (diabetes, fallos renales, patologías respiratorias o estado inmunocomprometido) (Joseph CA. 2010; Straus WL.1996).

Las superficies acuáticas constituyen el reservorio natural de esta bacteria, donde vive en simbiosis con protozoos de vida libre (amebas), que contribuyen a su replicación y difusión a nuevos ambientes o mayoritariamente en redes de agua de consumo asociada con comunidades bacterianas formando biopelículas. En función de la probabilidad de dispersar este biocontaminante, la legislación española clasifica las fuentes en instalaciones de alto y bajo riesgo (MISACO, 2003). Los sistemas domiciliarios de agua de consumo han sido referidos en estudios científicos como posible fuente de infección, especialmente si el sistema no es utilizado durante largos períodos, o tienen problemas de mantenimiento o relacionados con el control de la temperatura (Borella P, et al. 2004).

En Andalucía corresponde a la Consejería de Igualdad, Salud y Políticas Sociales del Gobierno regional, la inspección, evaluación y coordinación de las actuaciones que procedan ante la aparición de casos esporádicos de legionelosis (Junta de Andalucía, 1996). Los Distritos de Atención Primaria del Servicio Andaluz de Salud tienen entre sus cometidos desarrollar el programa de salud ambiental sobre prevención de la legionelosis (Junta de Andalucía, 2002), que incluye la intervención ambiental ante la aparición de casos aislados y/o brotes, señalando las instalaciones sospechosas de ser el origen de la infección y la adopción de medidas de control para evitar o reducir el riesgo de propagación de la enfermedad.

L. pneumophila es una importante causa de brotes epidémicos aunque la mayoría de los casos comunitarios se consideran esporádicos, y raramente se llega a conocer la fuente de infección. Probablemente por este motivo, los casos esporádicos reciben mucha menor atención en la literatura científica que los brotes, sin embargo, la existencia de casos esporádicos ayuda a describir la evolución de la incidencia de la enfermedad y a identificar patrones en una zona geográfica determinada.

El propósito de este estudio fue examinar la información sobre casos esporádicos del sistema de vigilancia de la legionelosis comunitaria en la ciudad de Córdoba (España) como recurso preventivo para la toma de decisiones en salud ambiental. Un segundo objetivo fue describir las implicaciones en salud pública de la investigación ambiental de casos esporádicos por dicha enfermedad.

\section{MÉTODO}

Definición de caso. Un caso esporádico comunitario de legionelosis es aquel que contrae la enfermedad fuera del ámbito hospitalario y sin que tenga relación epidemiológica con ningún otro caso. Definición clínica: enfermedad respiratoria aguda con signos focales de neumonía, fiebre, cefalea y mialgias. Como fuente de información se utilizó la base de datos de enfermedades de declaración obligatoria del 
sistema de vigilancia epidemiológica de Andalucía (MISACO, 1995; Junta de Andalucía, 1996). El análisis de datos se acotó cronológicamente al período evolutivo 2005-2010, ambos inclusive, con emplazamiento en el municipio de Córdoba (España), recuperando los registros que contenían los casos esporádicos notificados. Se excluyeron las personas que viajaron continuamente fuera del municipio durante los 10 días antes de la enfermedad.

Investigación clínica. La enfermedad del Legionario se confirmó cuando se detectó el antígeno $\operatorname{Lp} 1$ en orina y fue compatible con la definición clínica de caso. La presencia del antígeno urinario se analizó con kit comercial de Binax Now ${ }^{\circledR}$ (Scarborough, Maine, USA), que consistió en un ensayo inmunocromatográfico de membrana para la detección cualitativa del antígeno soluble de Lp1 de los pacientes afectados por legionelosis. Dicho antígeno es detectable en orina hasta tres días después del inicio de los síntomas.

Cuando se notificó un caso, los pacientes eran entrevistados mediante un cuestionario epidemiológico que incluía aspectos demográficos, lugar de residencia, datos clínicos, diagnóstico, actividades realizadas y movilidad así como factores ambientales (exposición ocasional o habitual a aerosoles producidos por dispositivos o instalaciones). La obtención de datos del historial clínico se extendió a las dos semanas previas al inicio de la enfermedad, debido a que algunos pacientes con patologías subyacentes pueden requerir más tiempo para desarrollar los síntomas.

Investigación ambiental. Para determinar la fuente de transmisión más probable de la legionelosis, se llevó a cabo la inspección de instalaciones sospechosas, realizada por agentes del servicio de salud pública coordinados por un técnico de salud especialista en sanidad ambiental y en cooperación con el Ayuntamiento de Córdoba (Junta de Andalucía, 2005), observando los criterios contenidos en diferentes guías técnicas sobre prevención y control de la legionelosis (Marcó J, 2006; AETCYR 2007; Junta de Andalucía 2008). Los ítems de inspección incluyeron los siguientes factores de riesgo en las instalaciones:

1. Estructurales: origen del agua, materiales, localización, generación de aerosoles;

2. Mantenimiento: biocidas, contaminación, estado higiénico y mecánico, sistema de tratamiento;

3. Operacionales: temperatura del sistema y del agua, filtración y renovación.

En el plazo máximo previsto de una semana desde la notificación del caso, se localizó y visitó el domicilio de los pacientes así como todos los edificios o zonas públicas que tenían torres de refrigeración y condensadores evaporativos en un radio de 500 metros del domicilio de residencia, lugar de trabajo o itinerario habitual del paciente, utilizando como recurso el censo municipal de instalaciones. Posteriormente 
se incrementó el radio de acción hasta 1.000 y 1.500 m, después se identificaron otras fuentes potencialmente contaminadas, por ejemplo, lavados a presión de vehículos, spas, jacuzzis, riego por aspersión, sistemas contra incendios, fuentes ornamentales. Las muestras ambientales se tomaron a partir de suficientes elementos de prueba sobre la fuente sospechosa y fueron enviadas y analizadas en el Laboratorio Provincial de la Consejería de Salud.

Uno de los propósitos de la vigilancia ambiental es la intervención precoz para corregir situaciones de riesgo e implantar medidas de prevención y control apropiadas, poniendo énfasis en la reducción del tiempo de notificación de los casos declarados. A tal efecto se estimaron los tiempos de demora en la notificación calculando la diferencia entre los días transcurridos entre la fecha de inicio de síntomas, la declaración del caso y el comienzo de la investigación ambiental.

Los Sistemas de Información Geográfica (SIG) son una herramienta esencial de soporte para la toma de decisiones en salud pública que sirve para identificar fuentes potenciales de infección e indagar algún patrón espacial que indique zonas con mayor probabilidad de contraer la enfermedad (García-Fulgueiras A, et al. 2003). Para la gestión espacial de la información se utilizó una base de datos SIG transferidos a un mapa digital donde se geo-referenciaron los domicilios y/o lugares de trabajo de los casos para buscar su relación con fuentes potenciales de exposición a aerosoles.

En todo caso los servicios de salud pública respondieron coordinadamente utilizando protocolos normalizados de vigilancia sanitaria (Junta de Andalucía, 2003), para confirmar o refutar las asociaciones epidemiológicas y ambientales identificadas.

Análisis estadístico. La tasa de incidencia bruta (x100.000 habitantes) y ajustada por sexo se estimó calculando el número anual de casos esporádicos utilizando las cifras oficiales de población del Instituto Estadístico de Andalucía. La comparación entre valores medios de la edad de los grupos de casos se realizó con la prueba tstudent. El test exacto de Fisher se utilizó para valorar si la proporción de casos con factores de riesgo mostró diferencias significativas entre distintas áreas de la ciudad $(p<0,05)$. La información recopilada fue incorporada a una base de datos y fue analizada utilizando Microsoft Excel 2010 for Windows.

\section{RESULTADOS}

Un total de 31 casos esporádicos por enfermedad del Legionario se notificaron durante el período 2005-2010 en el municipio de Córdoba. La tasa de incidencia anual promedio fue 1,59 correspondiente a una media de 5,17 casos. La incidencia aumentó de 0,93 casos en 2005 hasta 1,22 al final del período, alcanzando en 2007 el mayor pico $(\mathrm{n}=3,4)$ (figura 1$)$. 
Figura 1. Tasa de Incidencia de la Legionelosis en Córdoba ( ${ }^{\circ}$ de casos) y España (España), 2005-2010.

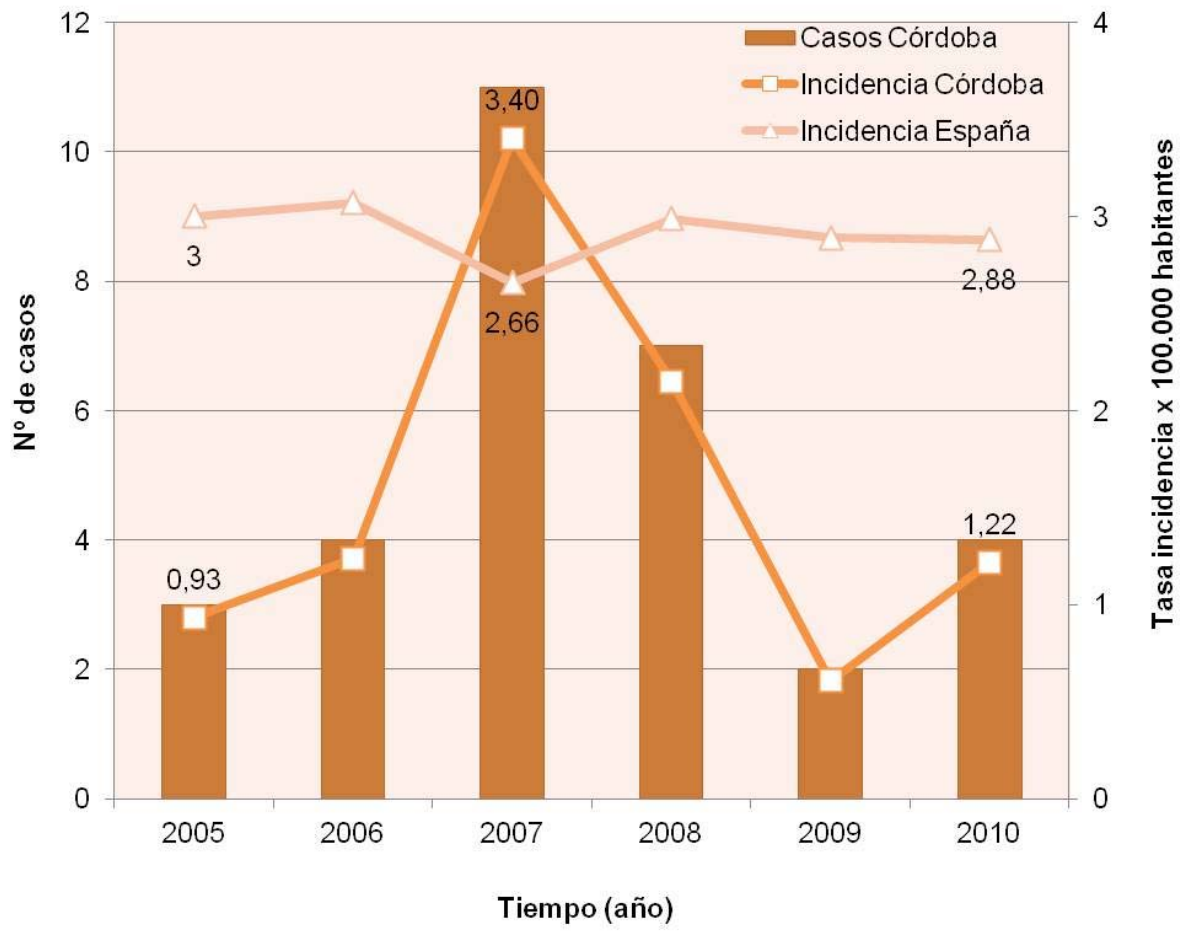

Fuente: Elaboración propia.

Características de los casos. Los 31 casos declarados dieron resultado positivo al test de antigenuria a Lp1, pero sin tipificación molecular del serogrupo. Edad: La edad media se situó en 56 $\pm 15,4$ años (rango:22-86), sin encontrar diferencias estadísticamente significativas $(\mathrm{t}=2,072 \mathrm{p}=0,06 \mathrm{IC}=99 \%)$ entre hombres $(\mathrm{n}=53 \pm 14,7)$ y mujeres $(n=65 \pm 14,4)$. Sexo: El sexo masculino representa el $74,2 \%$ de los pacientes con la enfermedad (figura 2). Factores de riesgo: El 70,97\% presentó algún factor de riesgo (tabla 1), en el 45,45\% existió una combinación de factores, mientras que en el 38,7\% alguna enfermedad subyacente. Se registró una víctima mortal en un paciente pluripatológico, sin determinarse si la causa fue por legionelosis. 
Figura 2. Casos esporádicos por sexo y grupos de edad en ámbito comunitario, Córdoba (España), 2005-2010. Fuente: Base datos EDO, Servicio Andaluz de Salud.

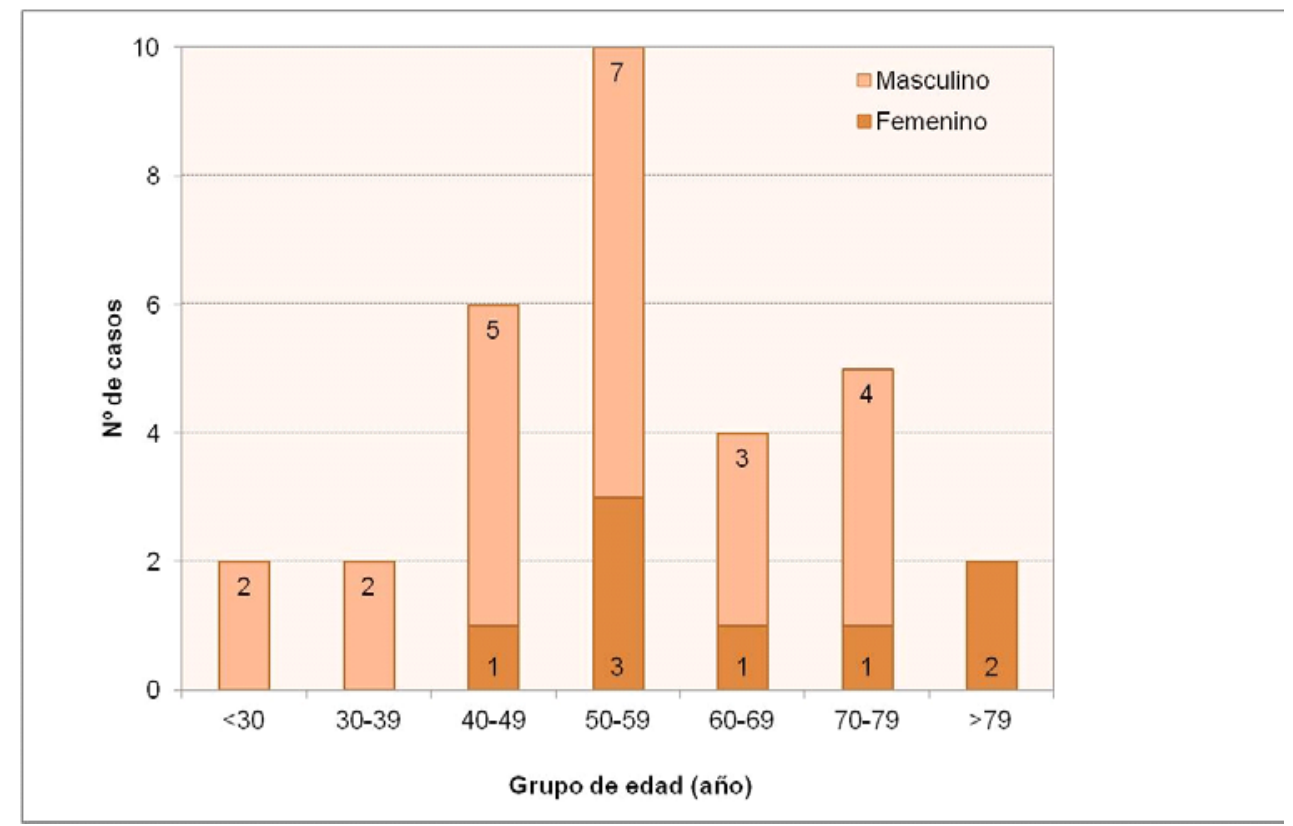

Fuente: Elaboración propia

Distribución espacio-temporal. Los casos se agruparon mayoritariamente en las estaciones de verano y otoño (35,48\% en cada período). El lugar de residencia de los pacientes estuvo disponible en el $96,78 \%$ de los casos. La mayoría de los pacientes residían y/o trabajaban en la ciudad de Córdoba (diámetro=4,5 Km). El 90,3\% de los casos no dejaron su lugar de residencia mientras que 3 casos realizaron visitas puntuales o de baja estancia fuera del municipio en las dos semanas previas al inicio de los síntomas.

Todos los casos esporádicos fueron visualizados en un mapa generado por SIG, observando que estaban repartidos de forma dispersa por el municipio. No obstante se identificaron dos zonas urbanas donde se concentraron, en un radio de 500 metros, el $19,35 \%$ y $22,58 \%$ de los casos del período (figura 3 ).

No se encontraron evidencias de que la edad media de los casos agrupados fuese significativamente diferente en una $(\mathrm{t}=0,656 \mathrm{p}=0,53)$ y otra zona identificada $(\mathrm{t}=0,356 \mathrm{p}=0,73)$, con respecto al resto del área urbana. Para valorar si dichas zonas presentaron una proporción diferente de casos mayores de 50 años con relación al resto de la ciudad, se aplicó el test exacto de Fisher, sin hallar diferencias significativas en ambas zonas $(p=0,135-0,334)$. 
Tabla 1. Características de los casos esporádicos comunitarios de legionelosis en Córdoba (España), 2005-2010.

\begin{tabular}{|c|c|c|c|}
\hline & $\mathbf{N}^{0}$ & Frecuencia & Rango edad (SD) \\
\hline \multicolumn{4}{|l|}{ Total pacientes } \\
\hline Total & 31 & 100 & \\
\hline Masculino & 23 & 74,2 & \\
\hline Femenino & 8 & 25,8 & \\
\hline \multicolumn{4}{|l|}{ Edad media } \\
\hline Total & 56 & & $22-86(15,37)$ \\
\hline Masculino & 53 & & $49-86(14,69)$ \\
\hline Femenino & 63 & & $22-75(14,37)$ \\
\hline Defunciones & 1 & 3,23 & \\
\hline \multicolumn{4}{|l|}{ Factores de riesgo } \\
\hline Fumador & 17 & 54,84 & \\
\hline Diabetes & 5 & 16,13 & \\
\hline Insuficiencia cardíaca & 3 & 9,68 & \\
\hline Inmunosupresión & 2 & 6,45 & \\
\hline Leucemia & 1 & 3,23 & \\
\hline $\mathrm{VIH}$ & 1 & 3,23 & \\
\hline Alcoholismo & 1 & 3,23 & \\
\hline
\end{tabular}

Fuente: Base de datos EDO. Servicio Andaluz de Salud.

Vigilancia ambiental. El censo municipal de instalaciones tuvo registradas un promedio de 130 unidades de torres de refrigeración y/o condensadores evaporativos distribuidas en 175 edificios.

Se identificaron un total de 40 torres en un radio menor de 500 metros del domicilio y/o lugar de trabajo del total de afectados. Se inspeccionaron 22 torres de refrigeración, 3 condensadores evaporativos, se localizaron 7 torres no registradas y 11 fuera de funcionamiento por parada estacional o avería. El 41,94\% de los pacientes residía a menos de 500 metros de una instalación con sistema de refrigeración evaporativo.

El $30 \%$ de las torres inspeccionadas presentaron deficiencias de mantenimiento; no obstante, los registros de análisis microbiológicos fueron negativos a Legionella spp. Se inspeccionaron el $41,94 \%$ de los suministros domésticos de agua en los domicilios de los pacientes, encontrando deficiencias estructurales en el 15,38\% de los casos (tabla 2). Como consecuencia de las intervenciones, las instalaciones inspeccionadas fueron vigiladas y se implantaron 
medidas correctoras, si bien tales actuaciones no eran el objeto de la investigación ambiental.

Figura 3. Mapa de distribución de casos esporádicos de legionelosis de ámbito comunitario, Córdoba 2005-2010

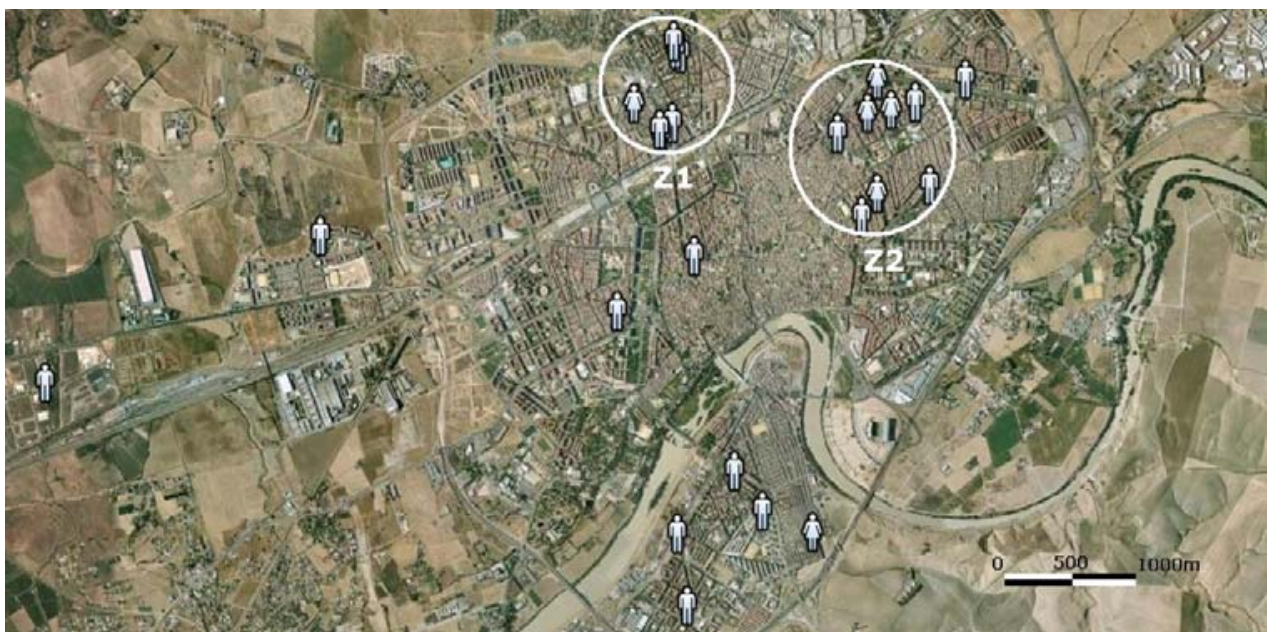

Fuente: Google Maps. Imagen TerraMetrics, Basarsoft. Orion-Me.

Tabla 2. Investigación de fuentes potenciales de contaminación por Legionella en Córdoba (España), 2005-2010.

\begin{tabular}{|c|c|c|c|c|c|c|}
\hline \multirow[b]{3}{*}{ Fuente ambiental potencial } & \multicolumn{4}{|c|}{ INSTALACIONES } & \multicolumn{2}{|c|}{ MUESTRAS } \\
\hline & & & $\mathrm{N}^{0}$ deficientes & & Medicion & n situ \\
\hline & Investigadas & Estructural & Mantenimiento & Operacional & Muestras & $\begin{array}{c}\text { No } \\
\text { cumplen }\end{array}$ \\
\hline
\end{tabular}

\begin{tabular}{|c|c|c|c|c|c|c|}
\hline Domicilio del paciente & 9 & 2 & & & 1 & \\
\hline Torre de refrigeración & 22 & 2 & 10 & & 3 & 1 \\
\hline Condensador evaporativo & 3 & & & & & \\
\hline Agua caliente sanitaria CR & 3 & & 3 & 1 & 13 & 8 \\
\hline Bañera hidromasaje (jacuzzi) & 2 & & & & & \\
\hline Piscina climatizada & 2 & & & & & \\
\hline Lavado automático vehículos & 1 & & & & & \\
\hline Fuente ornamental & 6 & & & & 2 & 2 \\
\hline Red municipal de riego jardines & 3 & & & & & \\
\hline $\mathrm{ACS} \sin \mathrm{CR}$ & 2 & & & & & \\
\hline Total & 53 & 4 & 13 & 1 & 19 & 11 \\
\hline
\end{tabular}

Fuente: Elaboración propia 
La combinación entre la información obtenida de la investigación ambiental y la fuente de datos epidemiológicos no indicó una única fuente sospechosa para la infección. Esta circunstancia unida a que sólo en 4 ocasiones se tomaron muestras ambientales para buscar evidencias microbiológicas de Legionella, impidió que pudiera identificarse la fuente de exposición.

Demoras en la notificación. El tiempo medio transcurrido entre la fecha de declaración de los casos y el inicio de la investigación ambiental fue de 7 días (rango: 0-93), a la que se añadió una demora acumulada de 27 días desde el inicio de los síntomas (rango:4-29).

\section{DISCUSIÓN}

Los datos del presente estudio muestran que el número de casos de legionelosis notificados en el municipio de Córdoba durante los años recientes se mantienen por debajo del promedio nacional. La tasa de incidencia de la enfermedad en la ciudad de Córdoba desde el año 2005 hasta el 2010 como promedio fue 2,5 veces menor comparada con la tasa nacional (CNE, 2010). De forma puntual, en el año 2007 encontramos un aumento de la tasa por encima de la media nacional. Este hallazgo puede reflejar una mayor concienciación en la notificación de casos a raíz de precedentes brotes de legionelosis con gran impacto mediático (Castilla J. et al, 2008), mayor sensibilidad del sistema de vigilancia, mejoras en la calidad de los datos del sistema de información, avances en el diagnóstico e investigación de los casos (Fields BS, et al. 2002) o incluso variaciones en las condiciones climáticas locales (Fisman $\mathrm{DN}$, et al.2005).

Aunque la percepción común es que la legionelosis es una enfermedad que afecta principalmente a los ancianos (Domínguez A, et al. 2009), la proporción de enfermos fue superior en el grupo de edad comprendido entre 50-60 años en todo el período. Se encontró una predominancia de varones que adquirieron la enfermedad y se identificaron otros factores ampliamente referidos en la literatura científica (Campese C, et al.2011; Den Boer JW, et al.2006; Marston BJ, et al.1994), como la presencia de enfermedades subyacentes (diabetes) y que más de la mitad de los casos mostraron estilos de vida (tabaquismo) con mayor riesgo de contraer legionelosis.

A pesar de los recursos desplegados y los esfuerzos llevados a cabo en la investigación multidisciplinar durante 5 años, no fue posible identificar una fuente de infección. Una razón elemental que explica este resultado radica en que no se realizaron muestreos ambientales exhaustivos para encontrar evidencias microbiológicas de Legionella, sino que el muestreo fue incompleto, no pudiendo así relacionar la fuente sospechosa de infección con la presencia de la bacteria en el ambiente. Además la configuración de la ciudad de Córdoba es compleja, por contar con múltiples fuentes productoras de aerosoles a las que podrían estar expuestos los pacientes en sus movimientos cotidianos, situación que hace difícil señalar a una única instalación como la principal fuente de infección.

Ciertamente el censo municipal facilitó la localización de instalaciones potencialmente contaminantes. En nuestra investigación ambiental se inspeccionaron 
suministros domiciliarios de agua, numerosas torres de refrigeración y una variada tipología de instalaciones en un radio de 500 metros alrededor de los domicilios de los enfermos, encontrando deficiencias estructurales y de mantenimiento en un considerable número de casos pero sin que llegara a conocerse la fuente común de infección.

No se cuestiona que focalizar la acción preventiva sobre las torres de refrigeración sea acertado, a tenor de las evidencias científicas que indican que dichas instalaciones juegan un importante papel en la causa de infecciones esporádicas (Che D, et al. 2003), pero esto puede ser infructuoso. Algunos autores (Stout JE, et al. 1987; 1992) cuestionan los trabajos que se centran en torres de refrigeración, demostrando que los suministros domiciliarios de agua pueden ser una fuente de infección por aerosoles, lo que plantea la conveniencia de explorar con mayor énfasis otras instalaciones susceptibles de dispersar Legionella. Esta oportunidad estaría justificada por la capacidad peculiar de supervivencia de la Legionella, invadiendo amebas o asociadas a biopelículas formadas en las superficies interiores de tuberías de agua, siendo así menos susceptible al efecto de los biocidas y causando serios problemas de salud pública (Borella $\mathrm{P}$, et al.2005).

La estrategia de prevención de la legionelosis debe tener como premisa un muestreo ambiental exhaustivo y rápido en el marco de una investigación completa, que sirva para confirmar o descartar fuentes potenciales de exposición y controlarlas, posibilitando conocer la importancia relativa del tipo de instalación involucrada y la carga de la enfermedad que lleva asociada cada fuente de infección. No se trata de emprender un "safari microbiológico" realizando muestreos sistemáticos y rutinarios sino más bien se precisa avanzar en la identificación de fuentes de exposición y, especialmente, ponderar la implicación de los diferentes tipos de instalaciones en la aparición de casos comunitarios. De esta manera se pretende no subestimar el problema para tener la oportunidad de evaluar el riesgo de legionelosis. A este respecto, la carga de trabajo generada a los servicios de salud pública, el tiempo invertido así como el coste implícito de los recursos empleados, son buenas razones para racionalizar las actuaciones conforme a criterios profesionales evitando que se realicen simplemente guiadas por criterios economicistas. En este sentido el costeutilidad de la vigilancia ambiental de Legionella ha sido ampliamente debatido ( $\mathrm{Yu}$ VL. 1998; Lock K, et al.2008).

El procedimiento de notificación de casos generó importantes demoras entre el tiempo en que se detectó cada caso comunitario y el momento en que se inició la vigilancia ambiental, dentro de lo que se concibe como un sistema de alerta temprana. Debe ponerse especial énfasis en la rapidez de la intervención al objeto de adoptar medidas efectivas de control y prevenir la aparición de casos (Jansà JM, et al. 2002). Por ello, una vez confirmados los casos, la notificación por medios digitales a tiemporeal entre los agentes involucrados debería reemplazar el sistema tradicional de comunicación interdepartamental.

La visualización del mapa SIG mostró dos zonas homogéneas dentro de un radio de $500 \mathrm{~m}$ donde se agruparon un llamativo número de casos esporádicos separados en el tiempo por más de seis meses, lo que soporta la hipótesis de que existen 
determinadas áreas geográficas donde el riesgo de exposición es mayor. Consideramos que estas dos zonas sospechosas tienen potencial interés porque el patrón mostrado es compatible con alguna fuente común de exposición desconocida o insuficientemente controlada y puede servir para valorar si el riesgo de aparición de nuevos casos de la enfermedad es relevante para justificar alguna forma temprana de intervención en salud pública que pueda ser efectiva.

La revisión de los sistemas de información de vigilancia de la legionelosis proporciona oportunidades para examinar las actuaciones de los servicios de salud pública y efectuar una puesta en común de conocimiento y recursos dirigida a mejorar los programas de salud ambiental sobre prevención de la legionelosis. La gestión espacio-temporal de los casos esporádicos permite reconocer a largo plazo zonas urbanas donde existen núcleos de población en riesgo por la presencia de instalaciones contaminadas por Legionella insuficientemente investigadas.

Las inspecciones ambientales no lograron identificar la fuente de infección, lo que indica que su capacidad predictiva del riesgo es muy limitada; por ello, los hallazgos de este estudio deberían alertar a las autoridades sanitarias para reorientar las estrategias preventivas, sobre todo teniendo en cuenta el importante despliegue de recursos humanos, materiales y los costes implícitos que conlleva la investigación de casos esporádicos.

\section{BIBLIOGRAFÍA}

ANDALUCÍA. Decreto 66/1996, de 13 de febrero, por el que se constituye, en la comunidad autónoma de Andalucía, el sistema de vigilancia epidemiológica y se determinan normas sobre el mismo. BOJA n ${ }^{\circ} 35$, de 19 de marzo.

ANDALUCÍA. Decreto 287/2002, de 26 de noviembre, por el que se establecen medidas para el control y la vigilancia higiénico-sanitarias de instalaciones de riesgo en la transmisión de la legionelosis y se crea el registro oficial de establecimientos y servicios biocidas de Andalucía. BOJA ${ }^{\circ} 144$, de 7 de diciembre.

ANDALUCÍA. Orden de 19 de diciembre de 1996, por la que se desarrolla el sistema de vigilancia epidemiológica en la comunidad autónoma de Andalucía y se establece la relación de enfermedades de declaración obligatoria. BOJA $n^{\circ} 4$, de 09 de enero.

ANDALUCÍA. Orden de 13 de octubre de 2005, por la que se delegan competencias para la suscripción de un Convenio entre la Consejera de Salud y el Ayuntamiento de Córdoba para el control de las instalaciones con riesgo de transmisión de legionelosis (torres de refrigeración y condensadores evaporativos). BOJA $\mathrm{n}^{\circ} 208$, de 25 de octubre.

ASOCIACIÓN TÉCNICA ESPAÑOLA DE CLIMATIZACIÓN Y REFRIGERACIÓN (2007). Guía técnica torres de refrigeración. Serie ahorro y eficiencia energética en climatización. Instituto para la Diversificación y el Ahorro de Energía. Madrid: Ministerio de Industria, Comercio y Turismo; 2007. 
BORELLA P, GUERRIERI E, MARCHESI I, BONDI M, MESSI P. (2005) Water ecology of Legionella and protozoan: environmental and public health perspectives. Biotechnol Annu Rev. 11:355-80.

BORELLA P, MONTAGNA MT, ROMANO-SPICE V et al. (2004) Legionella infection risk from domestic hot water. Emerg Infect Dis. 10(3):457-64.

CAMPESE C, BITAR D, JARRAUD S et al.(2011) Progress in the surveillance and control of Legionella infection in France, 1998-2008. Int J Infect Dis. 15:e30-e37.

CANO R, MARTÍN C, MANGAS I, MATEO S. (2001) Situación epidemiológica de la legionelosis en España. Gac Sanit. 15(2):76-ss.

CASTILlA J, BARRICARTE A, ALDAZ J et al. (2008) A large legionnaires' disease outbreak in Pamplona, Spain: early detection, rapid control and no case fatality. Epidemiol Infect.136: 823-32.

CENTRO NACIONAL DE EPIDEMIOLOGÍA (2010). Legionelosis. Situación general y evolución de la enfermedad en España. Datos de la Red Nacional de Vigilancia Epidemiológica (RENAVE). Madrid: Ministerio de Ciencia e Innovación; 2010.

CHE D, DECLUDT B, CAMPESE C, DESENCLOS JC. (2003) Sporadic cases of community acquired legionnaires' disease: an ecological study to identify new sources of contamination. J Epidemiol Community Health 57:466-9.

CEE. DG SANCO (2004). Health and the environment in the WHO european region: situation and policy at the beginning of 21 st century. EURO/04/5046267/14. Brussels: European Commission; 2004.

DEN BOER JW, NIJHOF J, FRIESEMA I. (2006) Risk factors for sporadic community-acquired legionnaires' disease. A 3-year national case-control study. Public Health 120:566-71.

DIRECCIÓN GENERAL DE SALUD PÚBLICA Y PARTICIPACIÓN. (2003) Sistemas de vigilancia epidemiológica de Andalucía: protocolos de alerta epidemiológica. Sevilla: Junta de Andalucía; 2003.

DOMÍNGUEZ A, ÁLVAREZ J, SABRIA M et al. (2009) Factors influencing the case-fatality rate of legionnaires' disease. Int J Tuberc Lung Dis. 13:407-12.

ESPAÑA. Real Decreto 865/2003, de 4 de julio, por el que se establecen los criterios higiénico-sanitarios para la prevención y control de la legionelosis. $\mathrm{BOE} \mathrm{n}^{\circ} 171$, de 18 de julio.

ESPAÑA. Real Decreto 2210/1995, de 28 de diciembre, por el que se crea la red nacional de vigilancia epidemiológica y según los criterios incluidos en los protocolos de dicha red. $\mathrm{BOE} \mathrm{n}^{\circ} 21$, de 24 de enero.

FIELDS BS, BENSON RF, BESSER RE. (2002) Legionella and Legionnaires' disease: 25 years of investigation. Clin Microbiol Rev.15(3): 506-526.

FISMAN DN, LIM S, WELLENIUS GA et al. (2005) It's Not the Heat, It's the Humidity: Wet Weather Increases Legionellosis Risk in the Greater Philadelphia Metropolitan Area. JID 192: 2066-72.

GARCÍA-FULGUEIRAS A, NAVARRO C, FENOLL D, GARCÍA J, GONZÁLEZDIEGO P, JIMÉNEZ-BUÑUALES T, et al. (2003) Legionnaires' disease outbreak in Murcia, Spain. Emerg Infect Dis. 9: 915-21. 
JANSÀ JM, CAYLÀ JA, FERRER D et al. (2002) An outbreak of legionnaires' disease in an inner city district: importance of the first 24 hours in the investigation. Int J Tuberc Lung Dis. 6(9):831-38.

JOSEPH CA, RICKETTS KD. (2010) Legionnaires' disease in Europe 2007-2008. Euro Surveill. 15(8):pii=19493.

LOCK K, MILLET C, HEATHCOCK R, JOSEPH CA, HARRISON TG, LEE JV, RAO G, SURMAN-LEE S. (2008) Public health and economic costs of investigating a suspected outbreak of legionnaires' disease. Epidemiol Infect. 136:1306-14.

MARCÓ J, MARTÍ S, MARTÍN JV et al. (2006). Guía técnica para la prevención y control de la legionelosis en instalaciones. Madrid: Ministerio de Sanidad, Consumo y Política Social; 2006.

MARSTON BJ, LIPMAN HB, BREIMAN RF. (1994) Surveillance for legionnaires' disease: risk factors for morbidity and mortality. Arch Intern Med. 154:2417-22.

SECRETARÍA GENERAL DE SALUD PÚBLICA Y PARTICIPACIÓN. (2008) Legionelosis. Procesos integrados de salud pública. Sevilla: Junta de Andalucía; 2008.

STOUT JE, VICTOR L, YU MD, MURACA MS. (1987) Legionnaires' disease acquired within the homes of two patients: link to the home water supply. JAMA, 257: 1215-1217.

STOUT JE, YU VL, MURACA P, JOLY J, TROUP N, TOMPKINS LS. (1992) Potable water as a cause of sporadic cases of community-acquired legionnaires' disease. N Engl J Med. 326(3):151-5.

STRAUS WL, PLOUFFE JF, FILE TM JR, LIPMAN HB, HACKMAN BH, SALSTROM SJ et al. (1996) Risk factors for domestic acquisition of legionnaires' disease. Ohio legionnaires' disease group. Arch Intern Med. 156(15):1685-92.

YU VL. (1998) Resolving the controversy on environmental cultures for Legionella. Infect Control Hosp Epidemiol.19:893-897. 